\title{
Computed Tomography of the Chest, Abdomen and Pelvis without Contrast
}

National Cancer Institute

\section{Source}

National Cancer Institute. Computed Tomography of the Chest, Abdomen and Pelvis

without Contrast. NCI Thesaurus. Code C137900.

Computed tomography of the chest, abdomen and pelvis without the use of a contrast agent. 